\title{
Did the universe have a beginning?
}

\author{
Audrey Mithani, Alexander Vilenkin \\ Institute of Cosmology, Department of Physics and Astronomy, \\ Tufts University, Medford, MA 02155, USA \\ Email : vilenkin@cosmos.phy.tufts.edu
}

\begin{abstract}
We discuss three candidate scenarios which seem to allow the possibility that the universe could have existed forever with no initial singularity: eternal inflation, cyclic evolution, and the emergent universe. The first two of these scenarios are geodesically incomplete to the past, and thus cannot describe a universe without a beginning. The third, although it is stable with respect to classical perturbations, can collapse quantum mechanically, and therefore cannot have an eternal past.
\end{abstract}

\section{Introduction}

One of the most basic questions in cosmology is whether the universe had a beginning or has simply existed forever. It was addressed in the singularity theorems of Penrose and Hawking [1], with the conclusion that the initial singularity is not avoidable. These theorems rely on the strong energy condition and on certain assumptions about the global structure of spacetime.

There are, however, three popular scenarios which circumvent these theorems: eternal inflation, a cyclic universe, and an "emergent" universe which exists for eternity as a static seed before expanding. Here we shall argue that none of these scenarios can actually be past-eternal.

Inflation violates the strong energy condition, so the singularity theorems of Penrose and Hawking do not apply. Indeed, quantum fluctuations during inflation violate even the weak energy condition, so that singularity theorems assuming only the weak energy condition 2] do not apply either. A more general incompleteness theorem was proved recently [3] that does not rely on energy conditions or Einstein's equations. Instead, it states simply that past geodesics are incomplete provided that the expansion rate averaged along the geodesic is positive: $H_{a v}>0$. This is a much weaker condition, and should certainly apply to the past of any inflating region of spacetime. Therefore, although inflation may be eternal in the future, it cannot be extended indefinitely to the past.

Another possibility could be a universe which cycles through an infinite series of big bang followed by expansion, contraction into a crunch that transitions into the next big bang [4. A potential problem with such a cyclic universe 
is that the entropy must continue to increase through each cycle, leading to a "thermal death" of the universe. This can be avoided if the volume of the universe increases through each cycle as well, allowing the ratio $S / V$ to remain finite [5]. But if the volume continues to increase over each cycle, $H_{a v}>0$, meaning that the universe is past-incomplete.

We now turn to the emergent universe scenario, which will be our main focus in this paper.

\section{Emergent universe scenario}

In the emergent universe model, the universe is closed and static in the asymptotic past (recent work includes [6, 7, 8, 9, 10, for early work on oscillating models see [11]). Then $H_{a v}=0$ and the incompleteness theorem [3] does not apply. This universe can be thought of as a "cosmic egg" that exists forever until it breaks open to produce an expanding universe. In order for the model to be successful, two key features are necessary. First, the universe should be stable, so that quantum fluctuations will not push it to expansion or contraction. In addition, it should contain some mechanism to exit the stationary regime and begin inflation. One possible mechanism involves a massless scalar field $\phi$ in a potential $V(\phi)$ which is flat as $\phi \rightarrow-\infty$ but increases towards positive values of $\phi$. In the stationary regime the field "rolls" from $-\infty$ at a constant speed, $\dot{\phi}=$ const, but as it reaches the non-flat region of the potential, inflation begins 12 .

Graham et al. 10 recently proposed a simple emergent model featuring a closed universe $(k=+1)$ with a negative cosmological constant $(\Lambda<0)$ and a matter source which obeys $P=w \rho$, where $-1<w<-1 / 3$. Graham et al. point out that the matter source should not be a perfect fluid, since this would lead to instability from short-wavelength perturbations [10. One such material that fulfills this requirement is a network of domain walls, which has $w=-2 / 3$. Then the energy density is

$$
\rho(a)=\Lambda+\rho_{0} a^{-1}
$$

and the Friedmann equation for the scale factor $a$ has solutions of the form of a simple harmonic oscillator:

$$
a=\omega^{-1}\left(\gamma-\sqrt{\gamma^{2}-1} \cos (\omega t)\right),
$$

where

$$
\omega=\sqrt{\frac{8 \pi}{3} G|\Lambda|}
$$

and

$$
\gamma=\sqrt{\frac{2 \pi G \rho_{0}^{2}}{3|\Lambda|}} .
$$

In the special case where $\gamma=1$, the universe is static. Although this model is stable with respect to classical perturbations, we will see that there is a quantum instability [13, 14]. 


\subsection{Quantum mechanical collapse}

We consider the quantum theory for this system in the minisuperspace where the wave function of the universe $\psi$ depends only on the scale factor $a$. In the classical theory, the Hamiltonian is given by

$$
\mathcal{H}=-\frac{G}{3 \pi a}\left(p_{a}^{2}+U(a)\right),
$$

where

$$
p_{a}=-\frac{3 \pi}{2 G} a \dot{a}
$$

is the momentum conjugate to $a$ and the potential $U(a)$ is given by

$$
U(a)=\left(\frac{3 \pi}{2 G}\right)^{2} a^{2}\left(1-\frac{8 \pi G}{3} a^{2} \rho(a)\right)
$$

With the Hamiltonian constraint $\mathcal{H}=0$, enforcing zero total energy of the universe, we recover the oscillating universe solutions discussed in [10].

We quantize the theory by letting the momentum become the differential operator $p_{a} \rightarrow-i \frac{d}{d a}$ and replacing the Hamiltonian constraint with the WheelerDeWitt equation [15]

$$
\mathcal{H} \psi=0 \text {. }
$$

From the Hamiltonian in Eq. (5), the WDW equation becomes

$$
\left(-\frac{d^{2}}{d a^{2}}+U(a)\right) \psi(a)=0,
$$

with the potential from Eqs. (1) and (7). Note that in quantum theory the form of the potential (see Fig. 1) is no longer that of a harmonic oscillator. Instead,

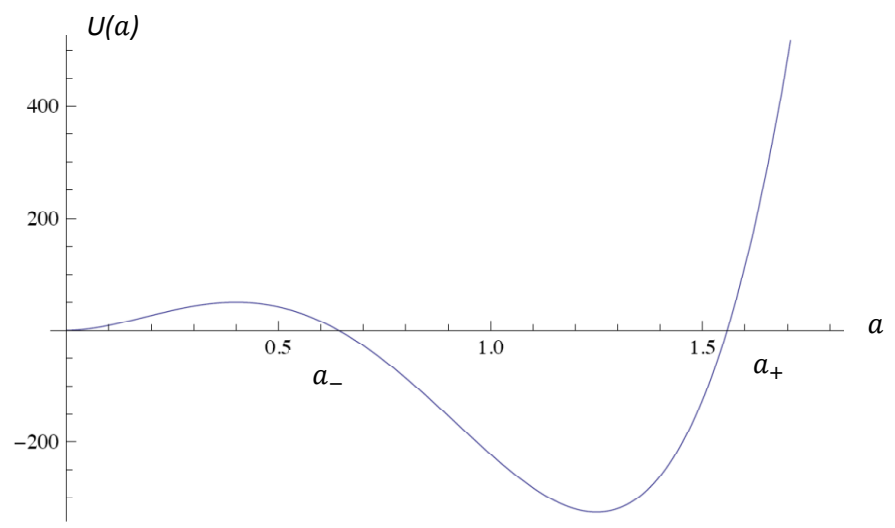

Figure 1: The potential $U(a)$ with turning points $a_{+}$and $a_{-}$ 
there is an oscillating region between the classical turning points $a_{+}$and $a_{-}$, which are given by

$$
a_{ \pm}=\omega^{-1}\left(\gamma \pm \sqrt{\gamma^{2}-1}\right)
$$

and the universe may tunnel through the classically forbidden region from $a_{-}$ to $a=0$. The semiclassical tunneling probability as the universe bounces at $a_{-}$ can be determined from 1

$$
\mathcal{P} \sim e^{-2 S_{W K B}}
$$

where the tunneling action is

$$
S_{W K B}=\int_{0}^{a_{-}} \sqrt{U(a)} d a=\frac{9 M_{P}^{4}}{16|\Lambda|}\left[\frac{\gamma^{2}}{2}+\frac{\gamma}{4}\left(\gamma^{2}-1\right) \ln \left(\frac{\gamma-1}{\gamma+1}\right)-\frac{1}{3}\right] .
$$

For a static universe, $\gamma=1$ and $a_{-}=a_{+}=\omega^{-1}$,

$$
S_{W K B}=\frac{3 M_{P}^{4}}{32|\Lambda|} .
$$

Since the tunneling probability is nonzero, the simple harmonic universe cannot last forever.

\subsection{Solving the WDW equation}

First let us examine the well-known quantum harmonic oscillator. In that case, the wave function is a solution to the Schrodinger equation

$$
\frac{1}{2}\left(-\frac{d^{2}}{d x^{2}}+\omega^{2} x^{2}\right) \psi(x)=E \psi(x) .
$$

After imposing the boundary conditions $\psi( \pm \infty) \rightarrow 0$, the solutions represent a discrete set of eigenfunctions, each having energy eigenvalue $E_{n}=\left(n+\frac{1}{2}\right) \omega$. However, in the case of the simple harmonic universe the wave function is a solution to the WDW equation (9), which has a fixed energy eigenvalue $E=0$ from the Hamiltonian constraint. From the form of the potential in Fig. 1, it seems that we must choose $\psi(\infty) \rightarrow 0$, so that the wave function is bounded at $a \rightarrow \infty$. We are then not free to impose any additional condition at $a=0$, or the system will be overdetermined. The wave function in the under-barrier region $0<a<a_{-}$is generally a superposition of growing and decaying solutions, and we can expect that the solution that grows towards $a=0$ will dominate (unless the parameters of the model are fine-tuned; see [14 for more details).

A numerical solution to the WDW equation is illustrated in Fig. 2, It exhibits an oscillatory behavior between the classical turning points and grows by magnitude towards $a=0$. This indicates a nonzero probability of collapse. Similar behavior is found for the case of $\gamma=1$, corresponding to a classically static universe.

\footnotetext{
${ }^{1}$ Semiclassical tunneling in oscillating universe models has been studied in the early work by Dabrowski and Larsen [13].
} 


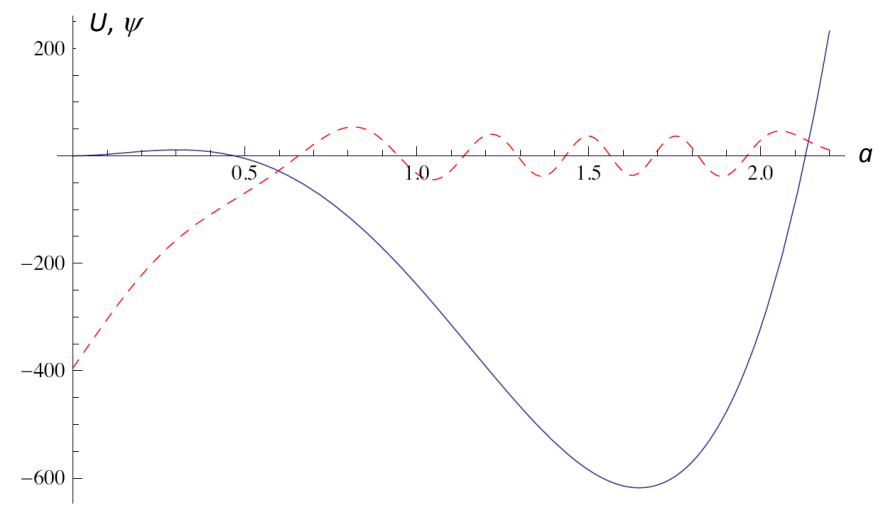

Figure 2: Solution of the WDW equation with $|\Lambda| / M_{P}^{4}=.028$ and $\gamma=1.3$ (dashed line). The WDW potential is also shown (solid line).

One can consider a more general class of models including strings, domain walls, dust, radiation, etc.,

$$
\rho(a)=\Lambda+\frac{C_{1}}{a}+\frac{C_{2}}{a^{2}}+\frac{C_{2}}{a^{3}}+\frac{C_{4}}{a^{4}}+\ldots
$$

For positive values of $C_{n}$, the effect of this is that the potential develops another classically allowed region at small $a$. So the tunneling will now be to that other region, but the qualitative conclusion about the quantum instability remains unchanged. Altering this conclusion would require rather drastic measures. For example, one could add a matter component $\rho_{n}(a)=C_{n} / a^{n}$ with $n \geq 6$ and $C_{n}<0$. Then the height of the barrier becomes infinite at $a \rightarrow 0$ and the tunneling action is divergent. Note, however, that such a negative-energy matter component is likely to introduce quantum instabilities of its own.

\section{Did the universe have a beginning?}

At this point, it seems that the answer to this question is probably yes ${ }^{2}$ Here we have addressed three scenarios which seemed to offer a way to avoid a beginning, and have found that none of them can actually be eternal in the past. Both eternal inflation and cyclic universe scenarios have $H_{a v}>0$, which means that they must be past-geodesically incomplete. We have also examined a simple emergent universe model, and concluded that it cannot escape quantum collapse. Even considering more general emergent universe models, there do not seem to be any matter sources that admit solutions that are immune to collapse.

\footnotetext{
${ }^{2}$ Note that we use the term "beginning" as being synonimous to past incompleteness.
} 


\section{References}

[1] S. W. Hawking and G. F. R. Ellis, The Large Scale Structure of Spacetime (Cambridge University Press, Cambridge, England, 1973).

[2] A. Borde and A. Vilenkin, Eternal inflation and the initial singularity, Phys. Rev. Lett. 72, 3305 (1994) gr-qc/9312022.

[3] A. Borde, A.H. Guth and A. Vilenkin, Inflationary Spacetimes Are Incomplete in Past Directions, Phys. Rev. Lett. 90, 151301 (2003) grqc/0110012.

[4] P. J. Steinhardt and N. Turok, A cyclic model of the universe, Science 296, 1436 (2002) hep-th/0111030.

[5] R.C. Tolman, Relativity, Thermodynamics and Cosmology. Oxford University Press (1934).

[6] G.F.R. Ellis and R. Maartens, The emergent universe: inflationary cosmology with no singularity, Class. Quantum Grav. 21, 223 (2004) grqc/0211082 4 4].

[7] J.D. Barrow, G.F.R. Ellis, R. Maartens, and C.G. Tsagas, On the stability of the Einstein static universe, Class. Quant. Grav. 20, L155 (2003) grqc/0302094.

[8] S. del Campo, E. Guendelman, A.B. Kaganovich, R. Herrera and P. Labrana, Emergent universe from scale invariant two measures theory, Phys. Lett. B 699, 211 (2011), arXiv:1105.0651].

[9] P. Wu and H. Yu, Emergent universe from the Hor̆ava-Lifshitz gravity, Phys. Rev. D81, 103522 (2010) arXiv:0909.2821.

[10] P.W. Graham, B. Horn, S. Kachru, S. Rajendran, and G. Torroba, A Simple Harmonic Universe, arXiv:1109.0282.

[11] M. P. Dabrowski, Oscillating Friedmann cosmology, Ann. Phys. 248, 199 (1996) [gr-qc/9503017].

[12] D.J. Mulryne, R. Tavakol, J.E. Lidsey and G.F.R. Ellis, An emergent universe from a loop, Phys. Rev. D71, 123512 (2005) astro-ph/0502589.

[13] M. P. Dabrowski and A. L. Larsen, Quantum tunneling effect in oscillating Friedmann cosmology, Phys. Rev. D52, 3424 (1995) gr-qc/9504025].

[14] A.T. Mithani and A. Vilenkin, Collapse of simple harmonic universe, JCAP 01 (2012) 028 [arXiv:1110.4096r4].

[15] B.S. DeWitt, Quantum Theory of Gravity. 1. The Canonical Theory, Phys. Rev. 160, 1113 (1967). 